\title{
Severe COVID-19 Pneumonia is Associated with Increased Plasma Immunoglobulin G Agonist Autoantibodies Targeting the 5-Hydroxytryptamine 2A Receptor
}

\author{
Mark B. Zimering ${ }^{1,2 *}$ \\ ${ }^{1}$ Veterans Affairs New Jersey Healthcare System, East Orange NJ, USA \\ ${ }^{2}$ Rutgers-Robert Wood Johnson Medical School, New Brunswick, NJ, USA
}

^Corresponding author: Mark B. Zimering, Medical Service (111); 385 Tremont Ave, East Orange, New Jersey-07018, Fax: 908 604 5249; Email: mark.zimering@va.gov Received: January 26, 2021; Accepted: January 29, 2021; Published: February 02, 2021

\begin{abstract}
Aims: To test whether plasma autoantibodies targeting the 5-hydroxytryptamine 2A receptor increase in COVID-19 infection; and to characterize the pharmacologic specificity, and signaling pathway activation occurring downstream of receptor binding in mouse neuroblastoma N2A cells and cell toxicity of the autoantibodies.

Methods: Plasma obtained from nineteen, older COVID-19 patients having mild or severe infection was subjected to protein-A affinity chromatography to obtain immunoglobulin G fraction. One-fortieth dilution of the protein-A eluate was tested for binding to a linear synthetic peptide QN.18 corresponding to the second extracellular loop of the human 5-hydroxytryptamine 2A receptor. Mouse neuroblastoma N2A cells were incubated with COVID-19 IgG autoantibodies in the presence or absence of selective inhibitors of G-protein coupled receptors, signaling pathway antagonists, or a novel decoy receptor peptide.

Results: 5-hydroxytryptamine 2A receptor autoantibody binding occurred in 17 of 19 (89\%) patients with acute COVID-19 infection and increased level was significantly correlated with increased severity of COVID-19 infection. The agonist autoantibodies mediated acute neurite retraction in mouse neuroblastoma cells by a mechanism involving Gq11/PLC/IP3R/Ca2+ activation and RhoA/Rho kinase pathway signaling occurring downstream of receptor binding which had pharmacologic specificity consistent with binding to the 5-HT2A receptor. A novel synthetic peptide 5-HT2AR fragment, SN..8, dose-dependently blocked autoantibody-induced neurotoxicity. The COVID-19 autoantibodies displayed acute toxicity in bovine pulmonary artery endothelial cells (stress fiber formation, contraction) and modulated proliferation in a manner consistent with known 'biased agonism' on the 5-HT2A receptor.
\end{abstract}

Conclusion: These data suggest that 5-HT2AR targeting autoantibodies are highly prevalent may contribute to pathophysiology in acute, severe COVID-19 infection.

Keywords: COVID-19 infection, 5-Hydroxytryptamine 2 A receptor, Inflammation, Neurotoxicity

\section{Introduction}

The SARS-Cov-2 virus mediates hyper-inflammation and dysregulated immunity leading to 'cytokine storm' [1]. Inflammation predisposes to hypercoagulability and human autopsy studies severe COVID-19 infection demonstrated widespread microvascular occlusion in the lung, liver, kidney, heart and brain [2]. Endothelial cells harbor angiotensin converting enzyme 2 (ACE2), the cellular receptor for SARS-Cov-2 virus entry [3] and the host response (to SARSCov-2 virus infection) in severely-affected persons is characterized by 'endotheliitis' [4]. Previously, we had reported increased circulating agonist IgG autoantibodies to the 5 -hydroxytryptamine $2 \mathrm{~A}$ receptor in subsets of diabetic microvascular disease or neurodegenerative disorders [5]. The autoantibodies promoted endothelial cell apoptosis and were neurotoxic in vitro [6,7]. Since the 5-hydroxytryptamine $2 \mathrm{~A}$ receptor is expressed on platelets, innate and adaptive immune cells $[8,9]$ and it was reported to mediate (in part) chronic inflammation in certain animal models of autoimmunity [10-12], here we tested whether agonist 5-hydroxytryptamine $2 \mathrm{~A}$ receptor IgG autoantibodies increase in COVID-19 infection in association with severe disease.

\section{Patients and Methods}

\section{Patients}

Total nineteen patients were either admitted to an acute medical floor or intensive care unit at the Veterans Affairs New Jersey Healthcare System (VANJHCS; East Orange, NJ) between April-June 2020 because of symptomatic COVID-19 infection or became COVID-19 
PCR positive while residing on a subacute VANJHCS rehabilitation or nursing home unit. Blood was drawn for testing and validation of a new Clinical Laboratory Service, COVID-19 antibody assay. Leftover, discard plasma was provided by the Clinical Laboratory Service (Dr. Cynthia Bowman) for the purposes of this research study. The study was reviewed by the local VANJHCS Investigational Review Board and determined to be exempt from informed consent requirement. Plasma samples were stored at- 20 degrees $\mathrm{C}$ prior to isolation of IgG autoantibodies.

\section{Patient 1}

A 73-old-man who experienced pneumonia, respiratory failure, renal failure requiring dialysis and weeks-long period of hyperinflammation (i.e. markedly elevated WBC) who died 3.5 months after admission. During his months-long hospitalization, he was not treated with any medication having antagonist activity on the 5-HT2A receptor.

\section{Patient 2}

A 62-year-old man with major depressive disorder, hypertension, HIV, cirrhosis, who experienced COVID-19 pneumonia without respiratory failure. He was treated with the selective 5-HT2A receptor antagonist mirtazapine (45 mg nightly) during a several months hospitalization for intermittent abdominal pain of unknown etiology. He was discharged in stable condition to a long-term facility.

\section{Patient 3}

An 86-year old man with prior CVA, hypertension, dementia, atrial flutter with rapid ventricular response, and congestive heart failure who experienced pneumonia and respiratory failure. The tachycardia responded to digoxin therapy. He was treated with convalescent plasma and discharged in stable condition to a subacute rehabilitation facility.

\section{Patient 4}

A 72-year-old man with prior history of cerebrovascular accident, type 2 diabetes mellitus and hypertension who experience a mild COVID-19 infection

\section{Patient 5}

A 74-year-old man with refractory hypertension, prior history of TIA , type 2 diabetes mellitus who presented with intermittent left arm weakness for 1 day. He was treated with intravenous fluids and discharged home in stable condition.

\section{Patient 6}

A 73-year old man with diabetes and dementia who experienced an asymptomatic COVID-19 infection while residing on a long-term VA nursing home unit.

\section{Methods}

\section{Protein-A Affinity Chromatography}

Protein-A chromatography was carried out as previously reported [6].

\section{Synthetic Peptides}

All peptides were synthesized at Lifetein Inc. (Hillsborough, NJ) and had $\geq 95 \%$ purity including QN..18 (QDDSLVFKEGSCLLADDN), SN.8 (SCLLADDN), QF.7 (QDDSLVF), and VC.7 (VFKEGSC). An additional control, a scrambled sequence of SN..8 having amino acid sequence LASNDCLD, (LD..8) consisted of the same amino acids as in SN..8, but arranged in a scrambled sequence.

\section{Enzyme Linked Immunosorbent Assay (ELISA)}

An enzyme linked immunosorbent assay employed 50 microgram per milliliter concentration of QN..18, which has an amino acid sequence corresponding to the second extracellular loop region of the human 5-HT2A receptor, as the solid-phase antigen. The ELISA was performed as previously reported [5].

\section{Mouse Neuroblastoma N2 Cells}

Mouse neuroblastoma N2A cells were cultured in DMEM with $10 \%$ fetal calf serum.

\section{N2A Mouse Neuroblastoma Cell Neurite Retraction Assay}

Quantitative determination of acute neurite retraction following the addition of COVID-19 plasma autoantibodies in the presence or absence of selective antagonists was carried out as previously reported [5].

\section{N2A Mouse Neuroblastoma Cell Survival Assay}

An MTT assay was used to assess mouse neuroblastoma cell survival following exposure to COVID-19 plasma autoantibodies; and was carried out as previously reported [5].

\section{Bovine Pulmonary Artery Endothelial Cells}

Bovine pulmonary artery endothelial cells (BPAE) were obtained from Sigma Chemical Co. and they were cultured in Medium 199 with $10 \%$ fetal calf serum.

\section{Endothelial Cell Survival Assay}

BPAE cells were plated in 96-well plates and incubated for 72 hours prior to the addition of a $1: 50^{\text {th }}$ dilution of the protein-A eluate fraction from COVID-19 or age-matched patients without COVID-19 infection. After 48 hours incubation at 37 degrees $\mathrm{C}$ in a $\mathrm{CO}_{2}$ incubator, endothelial cell survival (\% basal endothelial cell number) was determined using a colorimetric detection system as previously reported [6].

\section{Chemicals}

Chemicals were obtained from Sigma Chemical Co., Inc. (St Louis, MO) except YM-254890 obtained from Tocris (Mpls., MN) and SB204741 obtained from Focus Biomolecules (Plymouth Meeting, PA).

\section{Protein Determinations}

Protein assays were carried out as previously reported [5].

\section{Statistics}

Comparisons were made using unpaired Student's t-test; and Pearson's correlation coefficient. 


\section{Results}

\section{Clinical Characteristics and Autoantibody Prevalence}

The baseline clinical characteristics in the study patients are shown in Table 1. Mean age was $67.3 \pm 8.9$ years. Nearly all patients had one or more co-morbidities, essential hypertension and diabetes mellitus being the most common ones (Table 1). In an enzyme linked immunosorbent assay using the second extracellular loop of the human 5-hydroxytryptamine $2 \mathrm{~A}$ receptor as the solid phase antigen, seventeen of nineteen (89.5\%) COVID-19 patients tested positive for autoantibodies having significantly increased receptor peptide binding, i.e. $>0.06 \mathrm{AU}$ or higher. The mean level of binding in a $1 / 40^{\text {th }}$ dilution of the autoantibodies in each of nineteen COVID-19 patients tested was 0.123 , i.e. three-fold above background (0.04) absorbance level (Table 1).

\section{Clinical Outcomes}

Total ten of nineteen patients (53\%) experienced pneumonia and overall $37 \%$ experienced respiratory failure. Seven patients died during the inpatient hospitalization: five patients having COVID-19 pneumonia and two patients due to a primary gastrointestinal disorder, either severe worsening of alcoholic hepatitis or from urosepsis complicated by a gastrointestinal bleed. Four of nineteen patients (21\%) experienced end-stage renal disease as a manifestation of acute severe COVID-19 infection (Table 2).

\section{Plasma Autoantibody Binding to 5-HT2A Receptor Peptide}

Patients suffering with COVID-19 pneumonia, respiratory failure and the subset who progressed to death had highest autoantibody binding to the 5-HT2A receptor peptide (Figure 1). Respiratory

Table 1. Baseline clinical characteristics and autoantibody in the 19 Covid-19 patients

\begin{tabular}{|c|c|c|}
\hline Risk factor & Mean (SD) $(\mathrm{N}=19)$ & \\
\hline Age (years) & $67.3(8.9)$ & \\
\hline Body mass index $(\mathrm{kg} / \mathrm{m} 2)$ & $27.0(2.9)$ & \\
\hline 5-HT2AR peptide binding^ (AU) & $0.123(.06)$ & Normal range (0.04-0.054) \\
\hline Proportion w/AAB binding $>/=0.06$ & 17 of $19(89.5 \%)$ & \\
\hline \multicolumn{3}{|l|}{ Co-morbidities } \\
\hline Hypertension (\%) & 79 & \\
\hline Diabetes mellitus (\%) & 42 & \\
\hline Cardiac disease $(\%)$ & 37 & \\
\hline Stroke/TIA (\%) & 21 & \\
\hline Major depression (\%) & 16 & \\
\hline Schizophrenia (\%) & 11 & \\
\hline Dementia (\%) & 11 & \\
\hline Cancer (\%) & 21 & \\
\hline
\end{tabular}

AAB-autoantibody; TIA- transient ischemic attack; AU-absorbance units

$\wedge$ A $1 / 40^{\text {th }}$ dilution of the protein-A eluate fraction of plasma was incubated with QN...18 linear synthetic peptide corresponding to second extracellular loop region of the human 5-HT2A receptor as reported [5].

Table 2. Clinical manifestation and outcome in 19 Covid-19 patients

\begin{tabular}{|lc|}
\hline Pneumonia $(\mathrm{Y} / \mathrm{N})$ & $10 / 9(53 \%)$ \\
\hline GI symptoms $(\mathrm{Y} / \mathrm{N})$ & $7 / 12(37 \%)$ \\
\hline Respiratory failure $(\mathrm{Y} / \mathrm{N})$ & $7 / 12(37 \%)$ \\
\hline ESRD $(\mathrm{Y} / \mathrm{N})$ & $4 / 15(21 \%)$ \\
\hline Death $(\mathrm{Y} / \mathrm{N})$ & $7 / 12(37 \%)$ \\
\hline
\end{tabular}

GI-gastrointestinal; ESRD- end-stage-renal disease; Y-yes, N-no

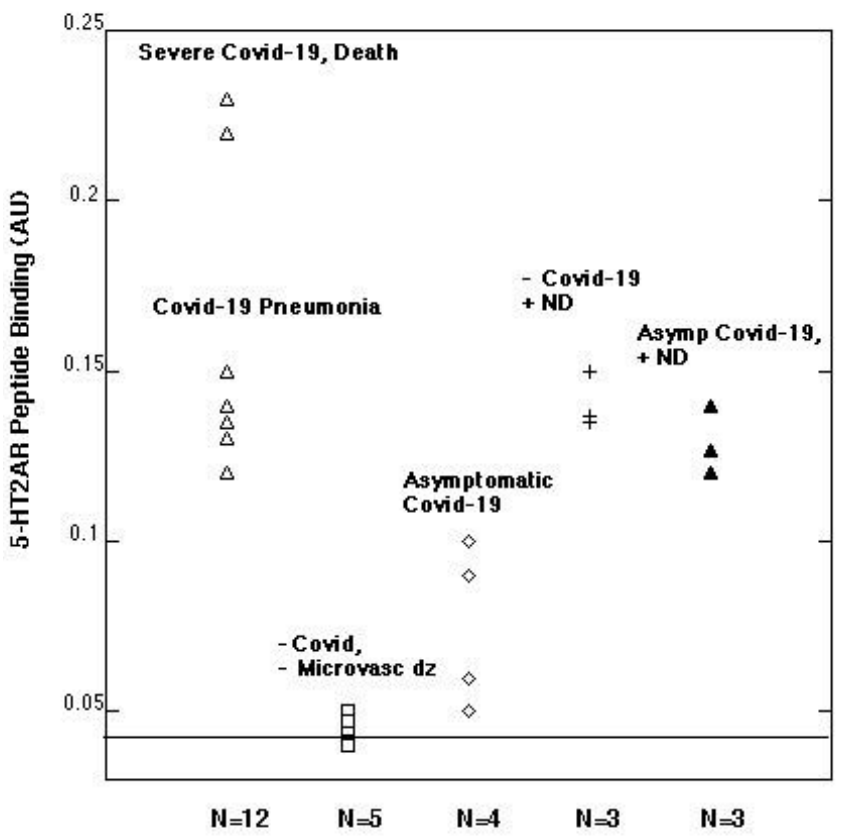

Figure 1: Plasma autoantibody binding to a linear synthetic 18-meric peptide QN...18 corresponding to the second extracellular loop of the human 5-hydroxytryptamine $2 \mathrm{~A}$ receptor.

A $1 / 40^{\text {th }}$ dilution of the protein-A eluate fraction of plasma was incubated with linear synthetic QN..18 peptide and binding was determined as previously reported [5]. NDneurodegenerative disease; microvasc(ular) dz-disease; Asymp(tomatic).

failure leading to death (in two patients) was associated with mean autoantibody binding level $(0.23 \mathrm{AU})$ more than 5.5-fold above background (0.04 AU) (Figure 1). COVID-19 with or without respiratory failure was associated with mean 3.25-fold increased autoantibody level compared to background (Figure 1). Persons with asymptomatic or minimally symptomatic COVID-19 infection $(n=4)$ had much lower level of autoantibody binding, (mean 0.08 AU, Figure 1). Plasma autoantibodies in age-matched patients without COVID-19 infection $(n=5)$ and not suffering from co-morbid vasculopathy or a neurodegenerative disorder(s) previously associated with elevated autoantibodies had no or nearly undetectable 5-HT2A receptor peptide binding (mean $0.04 \mathrm{AU}$, Figure 1).

\section{Role of Hyperinflammation in the De novo Appearance of COVID-19, 5-HT2AR Autoantibody}

A representative patient (Patient 1) who experienced multi-organ failure leading to death was a 73-year-old man who had a trajectory of white blood cell count level indicative of persistent hyperinflammation (Figure 2A). Plasma autoantibody binding to 5-HT2AR peptide was undetectable 5 days after the onset of symptoms, but (at day 35) had increased to 5.75 times higher than background level (Figure 2B). These are the first data to suggest de novo appearance of very high level of 5-HT2AR autoantibodies in association with hyperinflammation in severe COVID-19 infection.

\section{Pre-existing 5-HT2AR autoantibodies in patients having co- morbid neurodegenerative disease}

An IgG immune response to the COVID-19 virus spike protein was reported to be present in essentially all patients tested more than 
A)

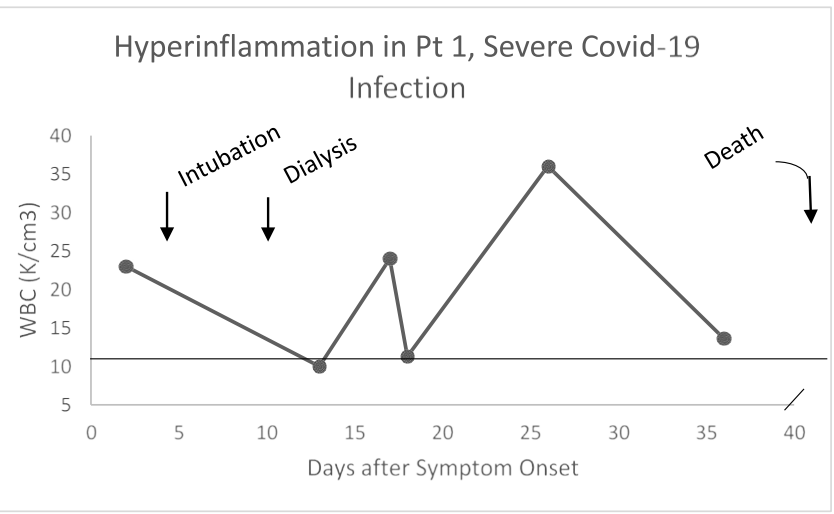

B)

Emergence of Plasma 5-HT2AR Autoantibodies in Pt 1 Severe Covid-19 infection

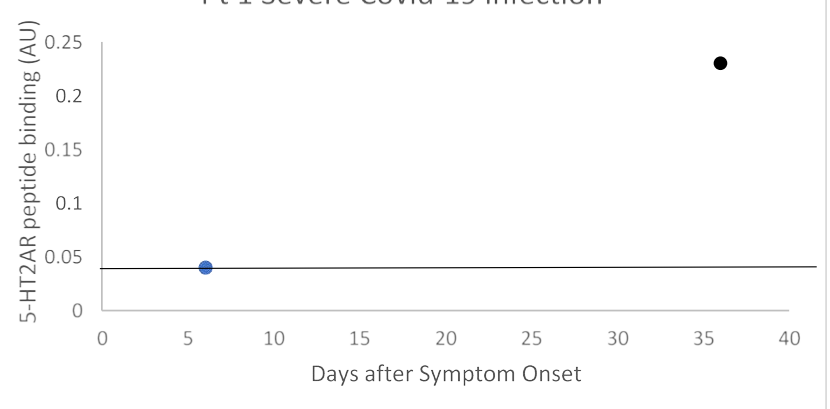

Figure 2: Clinical course (A) and de novo appearance of 5-HT2AR autoantibody (B) in plasma from a representative patient with severe Covid-19 pneumonia. Pt 1: A) 73-old-man who experienced pneumonia, respiratory failure, renal failure requiring dialysis and weeks-long period of hyperinflammation (i.e. markedly elevated WBC) who died 3.5 months after admission. B) Serotonin-2A receptor autoantibody binding was undetectable 6 days after hospital admission, but it had increased to 5.5 -fold greater than background level approximately 1 month later (on day 36). Dashed line (A) indicates upper limit of normal WBC, or (B) lower limit of detection of 5-HT2AR peptide binding.

10 days after the onset of clinical symptoms, but not earlier [13]. Yet three patients who experienced only mild COVID-19 symptoms (Figure 3A) already had substantially increased level of 5-HT2AR autoantibodies (mean 3-fold above background ) in blood drawn less than 5 days after symptom onset (Figure 3B). All three patients had a co-morbid neurodegenerative disorder, (i.e. stroke, refractory hypertension or dementia) previously reported to be associated with high level of 5-HT2AR-binding autoantibodies [5]. These data are consistent with preexisting 5-HT2AR autoantibodies which may not have increased substantially after mild COVID-19 infection.

\section{Correlation between Baseline Risk Factors, or Inflammation and 5-HT2AR Autoantibodies}

Consistent with a prior report [5] there was no significant correlation between age or body mass index and the level of 5-HT2AR autoantibody binding in plasma from nineteen COVID-19 patients tested (Figure 4A and 4B). After excluding four patients who had blood drawn for autoantibody determination $<5$ days after symptom onset, white blood cell count (a marker of systemic inflammation) was significantly correlated (Pearson correlation coefficient $\mathrm{R}=0.845 ; \mathrm{P}<$ 0.01 ) with 5-HT2AR autoantibody binding (Figure 5).
A

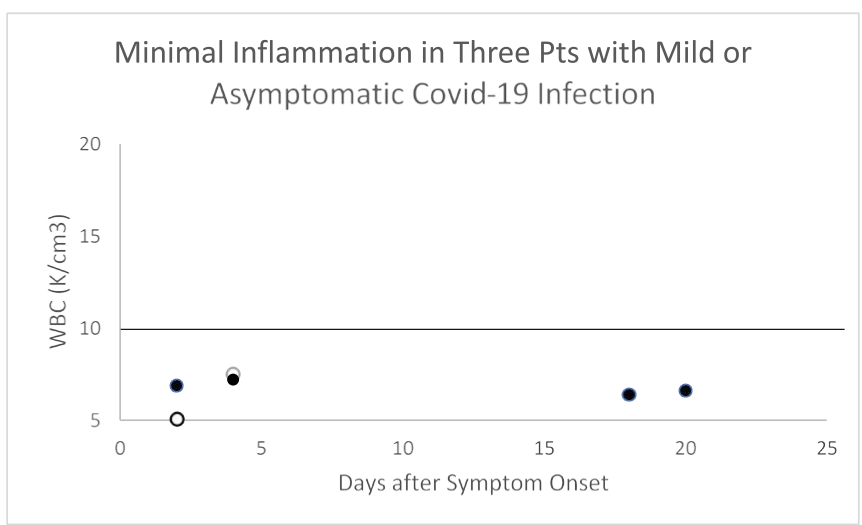

B

\section{Plasma 5-HT2AR Autoantibodies in Three Pts having mild Covid-19 infection}

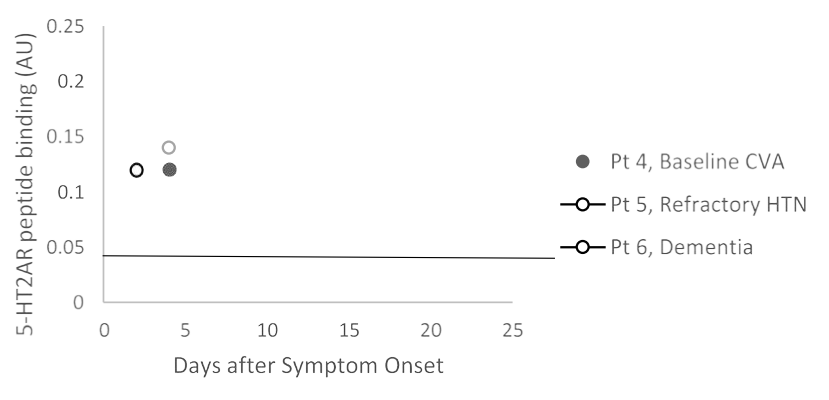

Figure 3: White blood cell count (A) and plasma 5-HT2AR autoantibodies (B) in three representative patients with asymptomatic Covid-19 infection who had co-morbid neurodegenerative disease. A) White blood cell counts in three patients having minimally symptomatic Covid-19 infection B) Increased 'preexisting' 5-HT2AR autoantibody binding manifested less than 1 week after onset of Covid-19 symptoms occurred in three patients having a co-morbid neurodegenerative conditions previously associated with high autoantibody level [5].

\section{Association between Plasma 5-HT2AR Autoantibodies and COVID-19 Disease Severity}

There was a gradient of increased plasma 5-HT2AR autoantibodies level for increasing severity of COVID-19 infection (Figure 6). Mean level of autoantibody binding in patients who experienced COVID-19 pneumonia, respiratory failure and death $(\mathrm{n}=5)$ was significantly higher ( 0.17 vs $0.08 ; \mathrm{P}<0.01)$ vs level in patients with mild or asymptomatic COVID-19 ( $n=4)$ (Figure 6). It was also significantly higher in patients who experienced COVID-19 pneumonia with or without respiratory failure $(\mathrm{n}=5)$ ( 0.13 vs $0.08 ; \mathrm{P}=0.02)$ vs those having only mild or asymptomatic infection $(\mathrm{n}=4)$ (Figure 6$)$. These data suggest a doseresponse relationship may exist between level of 5-HT2AR agonist autoantibodies and severity of COVID-19 infection consistent with a possible pathophysiologic role for COVID-19 disease autoantibodies. We next examined COVID-19, plasma 5-HT2AR peptide-binding autoantibodies for toxicity in neuroblastoma or endothelial cells.

\section{Neurotoxicity Associated with COVID-19 Disease 5-HT2AR Autoantibodies: Pharmacologic Profile}

In prior studies [6,7], plasma 5-HT2AR autoantibodies from patients having a neurodegenerative or microvascular disease caused acute 
Mark B. Zimering (2021) Severe COVID-19 Pneumonia is Associated with Increased Plasma Immunoglobulin G Agonist Autoantibodies Targeting the 5-Hydroxytryptamine 2A Receptor

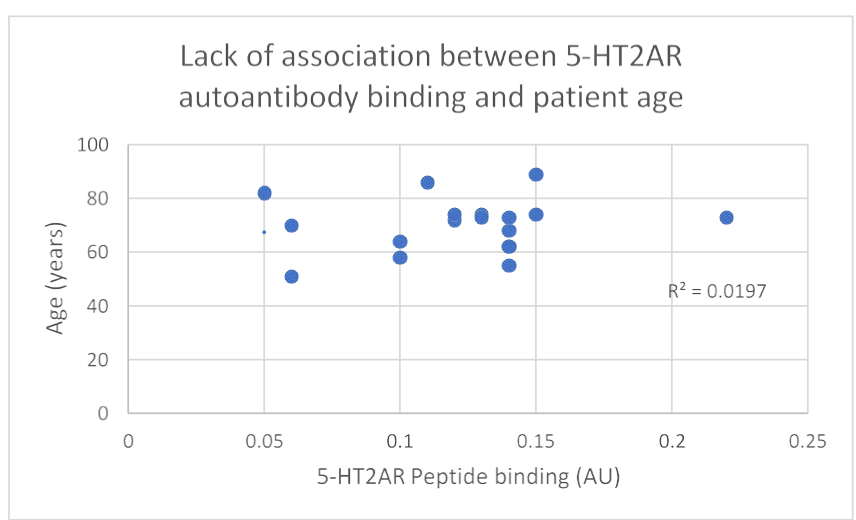

B

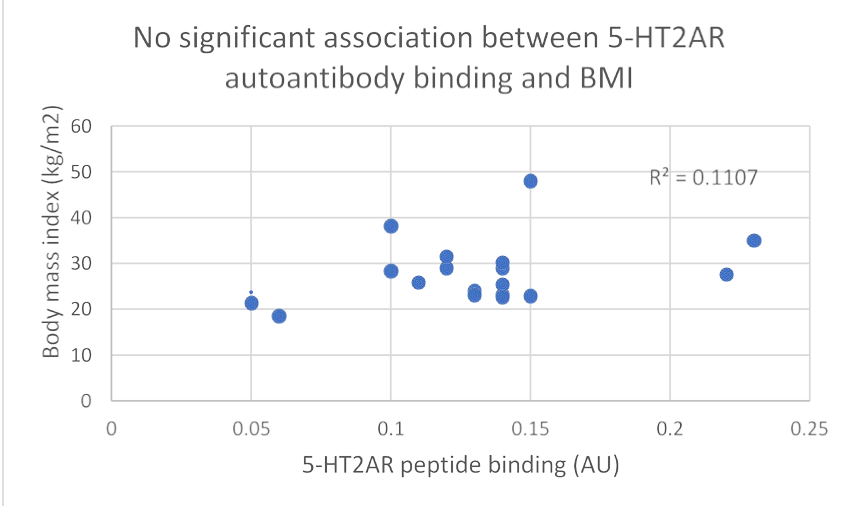

Figure 4: Lack of significant association between plasma 5-HT2AR binding autoantibodies and (A) age or body mass index (B) in 19 patients with Covid-19 infection. $\mathrm{N}=1$ patient had missing data on body mass index (BMI).

\section{Association between WBC and Covid-19 Plasma 5-HT2AR-targeting autoantibodies}

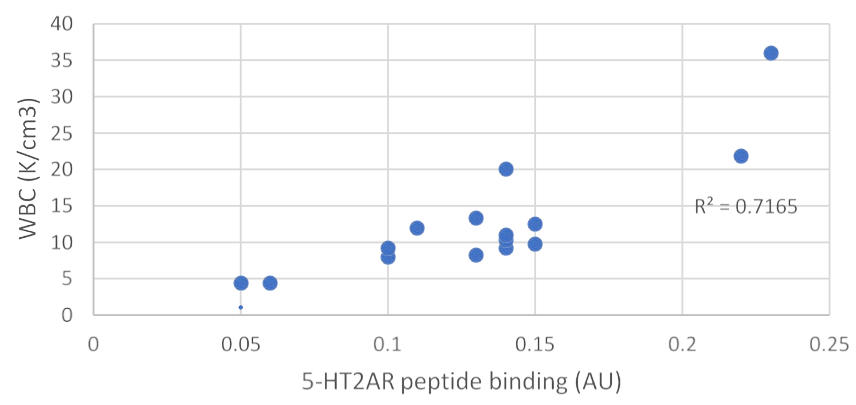

Figure 5: White blood cell count is significantly correlated with level of plasma autoantibodies to 5-HT2A receptor peptide in Covid-19 infection. Four patients were excluded from the analysis because blood drawing occurred less than 6 days after the initial onset of Covid-19 symptoms. Pearson correlation coefficient $(\mathrm{R}=0.845 ; \mathrm{P}<0.01$; $\mathrm{N}=15$ ).

neurite retraction and accelerated cell loss in mouse N2A neuroblastoma cells by a mechanism involving long-lasting activation of $\mathrm{Gq} / 11$ / phospholipase C/IP3R/Ca+2 signaling, and RhoA/Rho kinase activation. Here, COVID-19 autoantibodies caused acute neurite retraction (in mouse neuroblastoma cells) which was nearly completely prevented (95\%) by a 500 nanomolar concentration of the highly selective, potent 5-HT2AR antagonist M100907 (Table 3). COVID-19 autoantibodyinduced neurite retraction was also significantly prevented by a similar
5-HT2A receptor autoantibody binding correlates with Covid-19 pulmonary disease severity

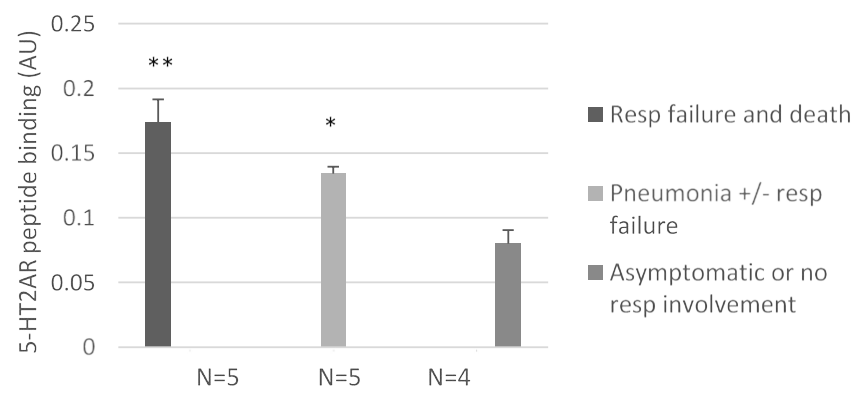

Figure 6: Association between severity of Covid-19 infection and level of plasma autoantibody binding to 5 -HT2AR peptide. ${ }^{* *} \mathrm{P}<0.01$ respiratory failure and death vs. asymptomatic or mild Covid-19 infection. ${ }^{*} \mathrm{P}=0.02$ pneumonia with or without respiratory failure vs mild Covid-19 infection. Results are mean (SD) of binding in a $1 / 40^{\text {th }}$ dilution of the protein-A eluate fraction of plasma.

or higher concentration of spiperone or ketanserin, antagonists that also have activity on the 5-HT2A receptor (Table 3). Specific antagonists of other classes of Gq/11-coupled G-protein coupled receptors, e.g. losartan, bosentan, prazosin, had much less (if any) protective effect on COVID-19 autoantibody-induced neurite retraction (Table 3). Taken together, the pharmacologic profile of neurotoxicity induced by COVID-19 autoantibodies is consistent with its binding to a linear synthetic peptide corresponding to the 5-HT2A receptor.

\section{Mechanism of Action of 5-HT2AR, COVID-19 Autoantibody Neurotoxicity}

Co-incubation of COVID-19 autoantibodies together with a specific antagonist of Gq/11 (YM-254890), phospholipase C (U73122), inositol triphosphate receptor (2-APB) or RhoA/Rho kinase (Y27632) signaling each completely abolished acute neurite retraction by the autoantibodies (Table 4). This suggests that COVID-19 autoantibody signaling downstream of 5-HT2AR receptor binding occurs via Gq11positively coupled to PLC/IP3R/Ca 2+ pathway activation and RhoA/ Rho kinase signaling consistent with the previously reported signaling pathways involvement in 5-HT2AR peptide-binding autoantibodies from patients (without COVID-19), but having a neurodegenerative disorder or diabetic microvascular angiopathy $[6,7]$.

Table 3. Pharmacologic profile of Covid-19 infection autoantibody-induced neurite retraction.

\begin{tabular}{|c|c|c|c|}
\hline Antagonist & {$[$ Conc] } & GPRC & $\begin{array}{l}\text { \% Inhibition of Covid-19 } \\
\text { AAb Neurite Retraction }\end{array}$ \\
\hline M100907 & $500 \mathrm{nM}$ & 5-HT2A/B/C & $95 \%$ \\
\hline Spiperone & $500 \mathrm{nM}$ & 5-HT2A/B/C & $72 \%$ \\
\hline Ketanserin & $5 \mu \mathrm{M}$ & $5-\mathrm{HT} 2 \mathrm{~A} / / \mathrm{B} / \mathrm{C}$ & $70 \%$ \\
\hline SB 204741 & $1 \mu \mathrm{M}$ & $5-\mathrm{HT} 2 \mathrm{~B}$ & $0 \%$ \\
\hline Losartan & $5 \mu \mathrm{M}$ & AT-1R & $30 \%$ \\
\hline Bosentan & $5 \mu \mathrm{M}$ & ET1-R & $10 \%$ \\
\hline Prazosin & $850 \mathrm{nM}$ & A1-AR & $20 \%$ \\
\hline
\end{tabular}

Results are (mean $+/-15 \%)$ on inhibition of N2A neurite retraction in 130 nanomolar concentration of severe Covid-19 autoantibody ( $\mathrm{Pt} 3$ ) by indicated concentration of each GPCR antagonist. AT-1R- angiotensin II, type 1 receptor; ET1-R-endothelin 1 receptor; A1-AR- alpha 1 adrenergic receptor; AAb- autoantibody 
Table 4. Effect of signaling pathway antagonists on Covid-19 autoantibody induced N2A neurite retraction

\begin{tabular}{|c|c|c|}
\hline Treatment & {$[$ Conc] } & $\begin{array}{c}\text { \% Covid-19 autoantibody-induced } \\
\text { neurite retraction }\end{array}$ \\
\hline YM-254890 (Gq/11 inhibitor) & $1 \mu \mathrm{M}$ & $0 \% \pm 0 \%$ \\
\hline 2-APB (IP3R inhibitor) & $20 \mu \mathrm{M}$ & $0 \% \pm 0 \%$ \\
\hline U73122 (PLC inhibitor) & $30 \mu \mathrm{M}$ & $0 \% \pm 0 \%$ \\
\hline Y27632 (ROCK inhibitor) & $10 \mu \mathrm{M}$ & $0 \% \pm 0 \%$ \\
\hline
\end{tabular}

Results are mean (SD) of two determinations on prevention of N2A neurite retraction in 130 nanomolar concentration of severe Covid-19 autoantibody (Patient 3) by the indicated concentration of pathway inhibitor.

\section{Receptor Decoy Peptide Prevents Neurotoxicity from COVID-19 Autoantibodies}

We next tested a receptor decoy peptide, SN..8, tentatively called 'Sertuercept' because it has amino acid sequence identical to an extracellular region of the serotonin 2 receptor ("Sertu") involved in mediating long-lasting receptor activation [14] and it may function as a decoy receptor ("ercept"). Sertuercept previously demonstrated neuroprotection against toxic effects of plasma 5-HT2AR autoantibodies from patients lacking COVID-19, but having either diabetic vasculopathy or a neurodegenerative disease [5]. Here, coincubation of COVID-19 autoantibodies together with increasing concentrations of Sertuercept dose-dependently prevented acute N2a cell neurite retraction; Sertuercept had $\mathrm{IC}_{50}$ of approximately 4 micromolar for half-maximal prevention of COVID-19 autoantibodyinduced acute N2A neurite retraction (Figure 7). Ten micromolar concentration of Sertuercept afforded $87.5 \%$ protection against neurite retraction induced by a 130 nanomolar concentration of COVID-19 autoantibodies from Patient 2 (Figure 8). An identical (10 uM) concentration of scrambled peptide sequence LN..8 having the same amino acids as in Sertuercept or higher $(20 \mathrm{uM})$ concentration of two peptides (e.g. QF..7 or VC..7) comprising adjacent subregions in the QN..18 sequence which comprises the second extracellular loop of the human 5-HT2A receptor did not significantly prevent COVID-19 autoantibody-induced neurite retraction (Figure 8 ). These data suggest that neuroprotection against COVID-19 autoantibody-induced toxicity is specific for the SN..8 peptide (Sertuercept) amino acid sequence.

Dose-dependent inhibition of Covid-19 autoantibody neurite retraction by SN...8 peptide

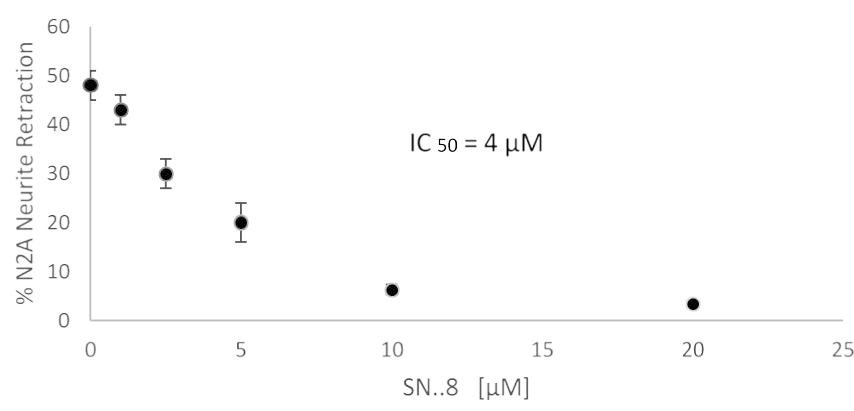

Figure 7: Dose-dependent inhibition of Covid-19 autoantibody induced N2A neuroblastoma cell neurite retraction by synthetic 5-HT2A receptor peptide fragment, SN..8. Mouse neuroblastoma N2A cells were incubated together with a 130 nanomolar concentration of autoantibody from a Covid-19 pneumonia patient (Patient 2) in the presence of the indicated concentration of 5-HT2A receptor peptide fragment SN..8. Acute neurite retraction was determined after 5 minutes as described in Methods.

\section{Inhibition of Covid-19 autoantibody-induced neurotoxicity by an SN..8 peptide}

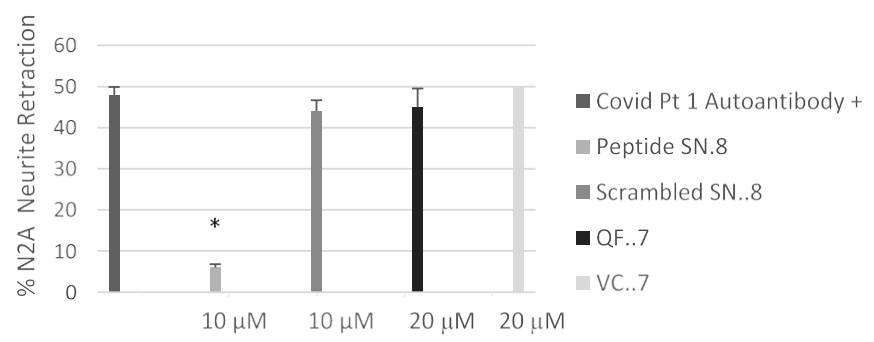

Figure 8: Specificity of 5-HT2A receptor peptide fragment SN. 8-mediated prevention of Covid-19 autoantibody induced N2A neurite retraction. ${ }^{*} \mathrm{P}<0.01$ compared to Covid-19 Pt 1 autoantibody (130 nM concentration) alone

Mouse neuroblastoma N2A cells were incubated together with a 130 nanomolar concentration of autoantibody from a Covid-19 pneumonia patient in the presence of the indicated concentration of receptor peptide SN..8 or a scrambled sequence LD..8 or peptide (QN..7 or VC..7) corresponding to adjacent regions of QN..18 [5]. Acute neurite retraction was determined after 5 minutes as described in Methods.

\section{Titer and Neurotoxicity of COVID-19, Plasma 5-HT2AR Autoantibodies}

In eight patients having symptomatic COVID-19 disease, the mean titer of 5-HT2AR binding autoantibodies (determined in blood drawn on average 3.1 weeks after symptom onset) was $\sim 67$ nM IgG. Mean titer in two patients with either Alzheimer's dementia or Parkinson's disease (without COVID-19) was somewhat higher perhaps consistent with much longer duration of disease. Titer in symptomatic COVID-19 $(\mathrm{n}=8)$ or neurodegenerative disease $(\mathrm{n}=2)$ autoantibodies was significantly higher than in three uncomplicated diabetic patients without microvascular complications (Figure 9). Plasma autoantibodies from symptomatic COVID-19 disease $(n=3)$ caused dose-dependent accelerated loss in mouse neuroblastoma cell N2a cells which significantly exceeded N2A cell loss induced by autoantibodies (tested at identical dilutions) from patients without COVID-19 infection (Figure 10).

\section{Pharmacologic Specificity of the COVID-19, 5-HT2A Receptor Targeting Autoantibodies}

Three different antagonists having a relative order of their affinity constants (M100907< spiperone $<<$ ketanserin) on the 5 -HT2AR each caused dose-dependent inhibition of COVID-19 autoantibody induced acute $\mathrm{N} 2 \mathrm{~A}$ neurite retraction (Figure 11). The $\mathrm{IC}_{50}$ for M100907 on $130 \mathrm{nM}$ concentration of COVID-19 autoantibodies (Pt 2) was approximately $270 \mathrm{nM}$ (Figure 11A). Spiperone and ketanserin was each tested against a lower (38 nanomolar) concentration of more highly potent, COVID-19 (Pt 3) neurodegenerative diseases autoantibody. Spiperone had an IC50 for inhibition of autoantibodyinduced neurite retraction of $\sim 300 \mathrm{nM}$ (Figure 11B). Ketanserin had an $\mathrm{IC}_{50}$ of $\sim 1.5 \mu \mathrm{M}$ consistent with ketanserin having relatively weaker antagonism on the 5-HT2AR. Maximal concentrations of either spiperone or ketanserin afforded partial (72-77\%) protection against the potent, Pt 3 COVID-19, neurodegenerative diseases autoantibodies (Figure 11B and 11C). 
Increased titer of 5-HT2AR Autoantibodies in Covid-19 symptomatic infection

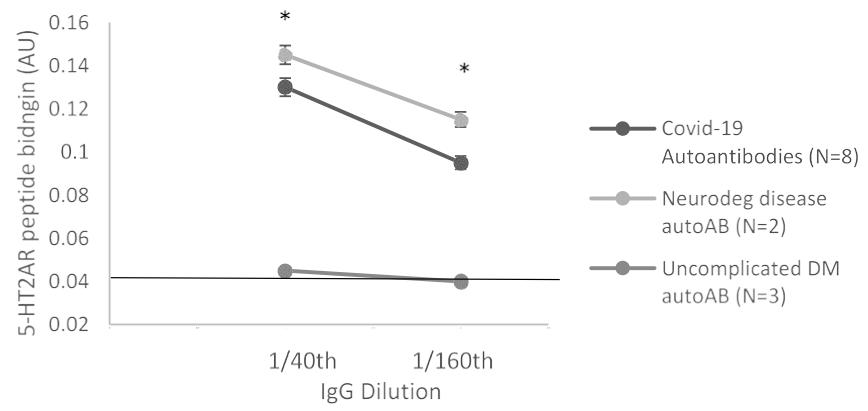

Figure 9: Increased titer of 5-HT2AR binding autoantibodies in plasma from eight Covid-19 patients: comparison to non-Covid patients having neurodegenerative disease or uncomplicated diabetes mellitus, ie. without vasculopathy or co-morbid neurodegeneration. ${ }^{*} \mathrm{P}<0.01$ vs binding in autoantibodies from uncomplicated $\mathrm{DM}$ diabetes mellitus, ie. without microvascular complications or neurodegenerative disorder.

Autoantibodies from Covid-19 patients, or non-Covid patients having either co-morbid neurodegenerative disorder or uncomplicated diabetes mellitus were tested for binding to QN..18 second extracellular loop of 5-HT2AR receptor peptide in ELISA. Covid-19 and non-Covid neurodegenerative disease autoantibodies displayed similarly high titer of autoantibody that exceeded binding in uncomplicated diabetes autoantibodies at each of two dilutions tested

\section{Dose-dependent neurotoxicity in symptomatic Covid-19 autoantibodies}

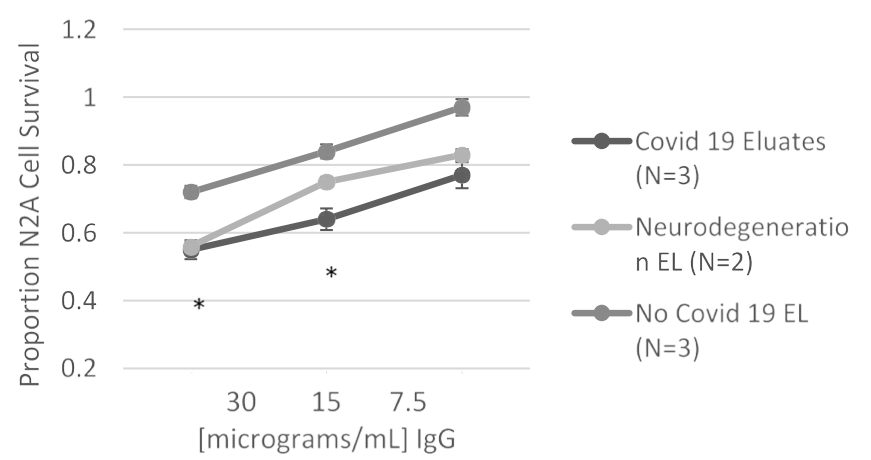

Figure 10: Covid-19 autoantibodies cause dose-dependent accelerated loss in mouse neuroblastoma N2a cells compared to patients without Covid-19 infection. ${ }^{*} \mathrm{P}<0.05$ comparing neurotoxicity in Covid-19 protein-A eluates to nearly identical concentration of protein-A eluate from patients without Covid 19

\section{Modulation of Endothelial Cell Survival by Autoantibodies in Severe COVID-19 Disease}

Mean endothelial cell survival was significantly decreased (66 \pm $18.5 \%, \mathrm{n}=4$ vs. $103.2 \pm 1.8 \% . \mathrm{n}=5 ; \mathrm{P}=0.003)$ after 2 days incubation with a $1 / 50^{\text {th }}$ dilution of the protein-A eluate from four COVID-19 plasmas compared to five age-matched patients without COVID-19 (Figure 12). Mean EC survival was significantly higher (114.5 $\pm 0.5 \%$, $\mathrm{n}=2$ vs. $103.2 \pm 1.8 \%, \mathrm{n}=5 ; \mathrm{P}<0.001)$ in the protein A eluates from two COVID-19 patients who had comorbid lymphoma or HIV disease compared to five patients without COVID-19 infection (Figure 10).

\section{COVID-19 Autoantibodies Cause Endothelial Cell Stress Fiber Formation and Acute Contraction}

Autoantibodies in patients having either diabetic microvascular complications [15] or a neurodegenerative disease [6,7] were
A

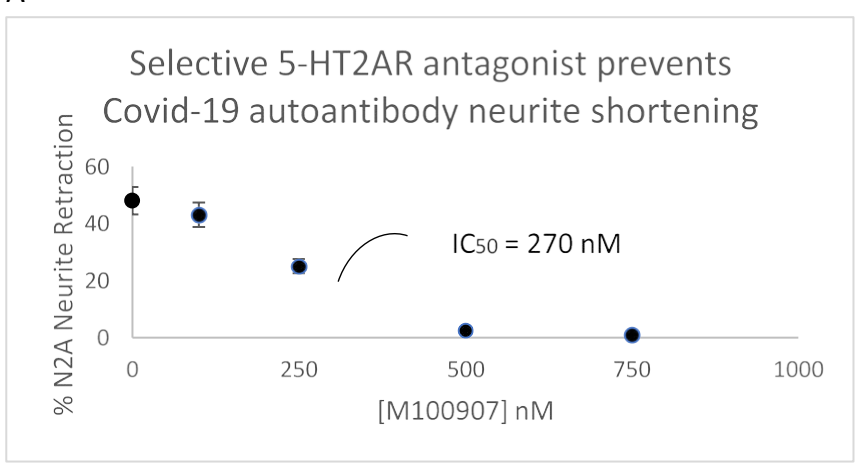

B

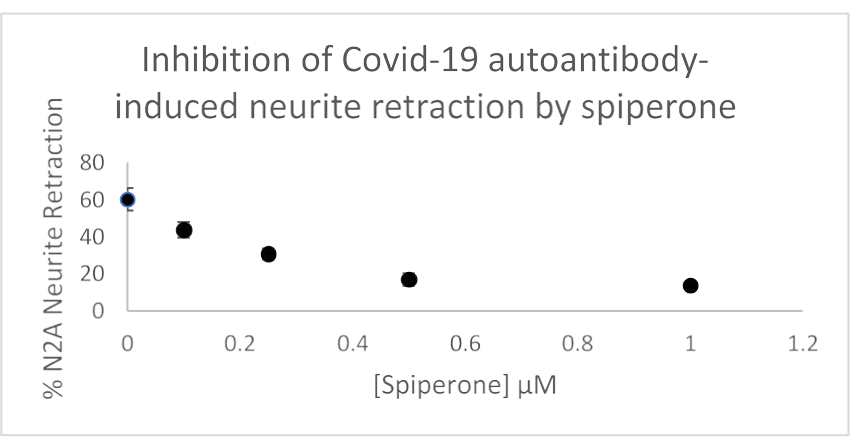

C

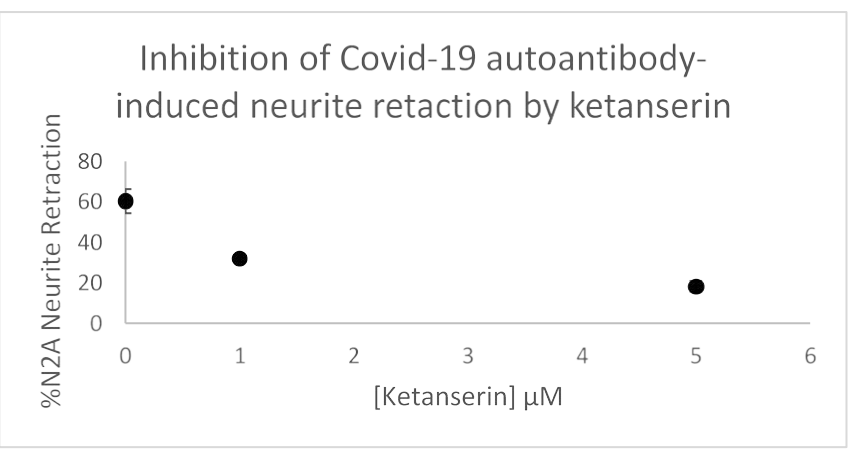

Figure 11: Dose-dependent prevention of Covid-19 autoantibody-induced N2a acute neurite retraction by three different 5-HT2AR antagonists: A) M100907, B) spiperone, C) ketanserin.

A) $130 \mathrm{nM}$ concentration of Patient 2 plasma autoantibodies; B-C) $38 \mathrm{nM}$ concentration of Patient 3 plasma autoantibodies.

previously reported to cause stress fiber formation and apoptosis in endothelial cells. In preliminary experiments, the Pt 3, COVID-19 autoantibodies (47 nanomolar concentration) caused stress fiber formation (within 5 minutes) and sustained contraction in bovine pulmonary artery endothelial cells (during 30 minutes continuous exposure). Pre-incubation with the receptor decoy peptide SN..8 (20 micromolar concentration) substantially prevented ( $80-90 \%)$ endothelial cell contraction induced by (twenty-eight nanomolar concentration) of the Pt 3, COVID-19 and dementia autoantibodies (data not shown).

\section{Discussion}

Severe COVID-19 infection causes pulmonary inflammation and diffuse endothelial cell dysfunction predisposing to multiorgan failure. The present data are the first to suggest that systemic 


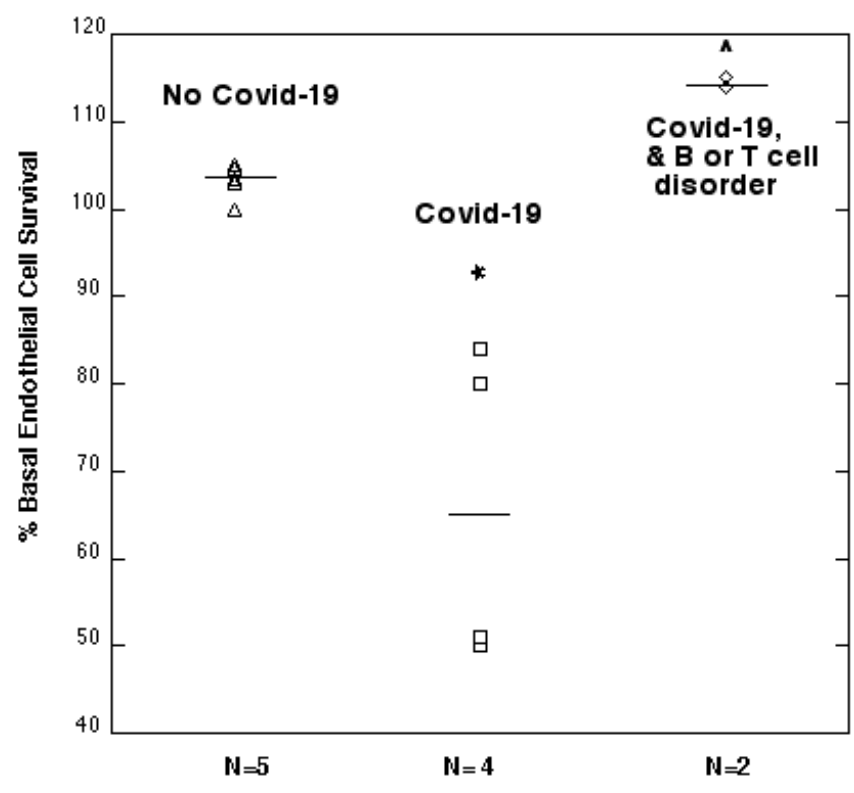

Figure 12: Modulation of endothelial cell survival by plasma autoantibodies from symptomatic Covid-19 infection: comparison to age-matched patients without Covid-19 infection.

${ }^{*} \mathrm{P}=0.003$ : Compared to EC survival in autoantibodies from patients with No Covid-19 infection.

$\wedge \mathrm{P}<0.001$ : Compared to EC survival in autoantibodies from patients with No Covid-19 infection.

inflammation in severe COVID-19 infection can give rise to the de novo appearance of very high level of IgG autoantibodies that specifically target the 5-HT2A receptor expressed on vascular endothelial cells and on neurons. Even though acute respiratory failure may occur prior to the emergence of IgG autoantibodies (in patients who lacked preexisting autoantibodies) a significant association between antibody level and severity of COVID-19 disease suggests a possible role (for the 5-HT2AR-targeting autoantibodies) in contributing to endothelial cell damage and/or neurotoxicity underlying ongoing disease pathophysiology.

Inflammation may have driven (in part) the appearance of 5-HT2AR-targeting autoantibodies in severe COVID-19 infection consistent with a prior report of a significant association between increased peripheral inflammation and 5-HT2AR autoantibodies in patients lacking COVID-19 infection, but having either obese type 2 diabetes mellitus or traumatic brain injury [16]. Angiotensin converting-enzyme 2, the cellular receptor for SARS-Co-V2 virus is abundantly expressed on endothelial cells [3] perhaps making certain antigens expressed on endothelial cells preferential targets of humoral immunity in SARS-Co-V2 viral infection. Endothelial cell inhibitory autoantibodies in patients having either diabetic vasculopathy or traumatic brain injury cross-reacted with heparan sulfate proteoglycan [16]. Anti-heparan sulfate proteoglycan autoantibodies occur in systemic lupus erythematosus and are thought to contribute to an increased risk of vascular thrombosis by interfering with the normal inhibitory effect of antithrombin III on thrombin [17]. Microvascular endothelial cell injury results in platelet adhesion and the 5-HT2A receptor which is expressed on platelets plays a role in platelet aggregation leading to 5 -HT (serotonin) release.
Recent autopsy studies in COVID-19 patients revealed diffuse microvascular occlusions in key organs including lung, liver, heart, kidney and brain [2]. High level of 5-HT2AR-targeting autoantibodies was previously reported [5] in patients without COVID-19 infection harboring retinal artery or retinal vein microvascular occlusion. The etiology of small and large vessel thromboses occurring in severe COVID-19infection is unknown and islikely to be multifactorial. Severe COVID-19 infection mimics aspects of systemic autoimmune disease including the presence of anti-phosphatidylserine autoantibodies implicated in causation of recurrent large vessel thrombosis e.g. anti-phospholipid syndrome [18] in systemic lupus erythematosus. For example, a recent study reported that approximately $25-50 \%$ of COVID-19 infected patients harbored either anti-phosphatidyl/ prothrombin antibodies or anti-phospholipid antibodies in the circulation [19]. Viral infections, certain cancers e.g. Burkitt's lymphoma [20] and systemic autoimmunity are all associated with an increased incidence of circulating immune complexes. The 5-HT2AR binding autoantibodies from two patients having COVID-19 infection and either co-morbid Burkitt's lymphoma or HIV disease caused significant endothelial cell proliferation consistent with a prior report of increased cell proliferation evoked by the 5-HT2AR-targeting autoantibodies from a patient with discoid lupus erythematosus [21]. The 5-HT2A receptor is known to mediate 'biased agonism' such that structural differences in the agonist can direct downstream signaling toward activation of beta arrestin 2-mediated survival pathways [22].

The 5-HT2AR is not only widely expressed in vascular tissue [23], but also in the central nervous system [24]. Previously, we reported that the highly potent, endothelial cell inhibitory plasma autoantibodies in a subset of cancer fatigue patients caused excitation followed by prolonged 'desensitization' of synaptic input in cultured rat hippocampal pyramidal neurons [25]. Fatigue and neurologic symptoms are among the most common manifestations of 'long haul' COVID-19, a syndrome in which various nonspecific symptoms can persist for weeks following recovery from acute COVID-19 infection. Longer term follow up in a diverse patient population is needed to test whether 5-HT2AR-targeting IgG autoantibodies may persist for weeks or months following acute COVID-19 infection and whether persistently elevated autoantibody level or titer may correlate with a subset of persistent 'long haul' COVID-19 symptoms.

Recently, we reported that use (vs non-use) in hospitalized COVID-19 infection of existing FDA-approved, 5-HT2AR antagonists (to treat comorbid neuropsychiatric illness or for ICU delirium) was associated with a significant, 5-fold lower odds ratio for mortality [26]. Based on the present data, one possibility is that 5-HT2AR antagonist medications block harmful effects from agonist 5-HT2AR autoantibodies expressed at high level in most cases of severe COVID-19 infection.

In summary, nearly ninety percent of patients with COVID-19 infection, many having pneumonia and requiring hospitalization, harbored substantial titer of neurotoxic and endothelial cell toxic plasma IgG autoantibodies which bound to a linear synthetic peptide corresponding to the second extracellular domain of the 5-HT2A receptor. Binding was associated with acute neurotoxicity which 
could be prevented (in vitro) either with specific 5-HT2A receptor antagonists or by a serotonin $2 \mathrm{~A}$ receptor peptide SN..8, Sertuercept, corresponding to a subregion important in mediating long-lasting 5-HT2A receptor activation [14]. Taken together, these data provide proof-of-principle that repurposing of existing FDA-approved 5-HT2AR antagonist medications or a novel decoy 5-HT2A receptor peptide (Sertuercept) might protect against harmful effects of 5-HT2A receptor agonist autoantibodies associated with COVID-19 infection.

\section{Acknowledgement}

Dr. Cynthia Bowman, Chief, Pathology and Laboratory Medicine Service, Veterans Affairs New Jersey Healthcare System (East Orange, New Jersey) for providing discard COVID-19 plasma samples used in the approved research study.

\section{References}

1. Buszko M, Park JH, Verthelyi D, Sen R, Young HA, Rosenberg AS (2020) The dynamic changes in cytokine responses in COVID-19: a snapshot of the current state of knowledge. Nat Immunol 21(10): 1146-1151.

2. Dolhnikoff M, Duarte-Neto AN, de Almeida Monteiro RA, da Silva LFF, de Oliveira EP, Saldiva PHN, Mauad T, Negri EM et al. (2020) Pathological evidence of pulmonary thrombotic phenomena in severe COVID-19. J Thromb Haemost 18(6): 1517-1519. [crossref]

3. Nascimento Conde J, Schutt WR, Gorbunova EE, Mackow ER, et al. (2020) Recombinant ACE2 Expression Is Required for SARS-CoV-2 To Infect Primary Human Endothelial Cells and Induce Inflammatory and Procoagulative Responses. mBio 11(6): e03185-20. [crossref]

4. Varga Z, Flammer AJ, Steiger P, Haberecker M, Andermatt R, Zinkernagel AS, Mehra MR, Schuepbach RA, Ruschitzka F, Moch H, et al. (2020) Endothelial cell infection and endotheliitis in COVID-19. Lancet 395: 1417-141. [crossref]

5. Zimering MB (2019) Autoantibodies in Type-2 Diabetes having Neurovascular Complications Bind to the Second Extracellular Loop of the 5-Hydroxytryptamine 2A Receptor. Endocrinol Diabetes Metab J 3(4): 118. [crossref]

6. Zimering MB (2017) Diabetes Autoantibodies Mediate Neural- and Endothelial CellInhibitory Effects Via 5-Hydroxytryptamine-2 Receptor Coupled to Phospholipase C/Inositol Triphosphate/Ca2+ Pathway. J Endocrinol Diabetes 4(4): 10.15226/23746890/4/4/00184. doi: 10.15226/2374-6890/4/4/00184. [crossref]

7. Zimering MB (2018) Circulating Neurotoxic 5-HT2A Receptor Agonist Autoantibodies in Adult Type 2 Diabetes with Parkinson's Disease. J Endocrinol Diabetes 5(2): 10.15226/2374-6890/5/2/01102. doi: 10.15226/2374-6890/5/2/01102.

8. Duerschmied D, Suidan GL, Demers M, Herr N, Carbo C, et al. (2013) Platelet serotonin promotes the recruitment of neutrophils to sites of acute inflammation in mice. Blood 121(6): 1008-1015. [crossref]

9. Herr N, Bode C, Duerschmied D (2017) The Effects of Serotonin in Immune Cells. Front Cardiovasc Med 4: 48. [crossref]

10. Wan M, Ding L, Wang D, Han J, Gao P (2020) Serotonin : A Potent Immune Cell Modulator in Autoimmune Diseases. Front Immunol 11: 186. [crossref]
11. Almishri W, Shaheen AA, Sharkey KA, Swain MG (2019) The Antidepressant Mirtazapine Inhibits Hepatic Innate Immune Networks to Attenuate ImmuneMediated Liver Injury in Mice. Front Immunol 10: 803. [crossref]

12. Xiao J, Shao L, Shen J, Jiang W, Feng Y, Zheng P, Liu F, et al. (2016) Effects of ketanserin on experimental colitis in mice and macrophage function. Int J Mol Med 37(3): 659-668. [crossref]

13. Graham NR, Whitaker AN, Strother CA, et al. (2020) Kinetics and isotype assessment of antibodies targeting the spike protein receptor-binding domain of severe acute respiratory syndrome-coronavirus-2 in COVID-19 patients as a function of age, biological sex and disease severity. Clin Transl Immunology 9(10): e1189. [crossref]

14. Wacker D, Wang S, McCorvy JD, Betz RM, Venkatakrishnan AJ, Levit A, Lansu K, Schools ZL, Che T, Nichols DE, Shoichet BK, Dror RO, Roth BL, et al. (2017) Crystal Structure of an LSD-Bound Human Serotonin Receptor. Cell 168(3): 377-389.e12. [crossref]

15. Zimering MB and Pan Z (2009) Autoantibodies in type 2 diabetes induce stress fiber formation and apoptosis in endothelial cells. J Clin Endocrinol Metab 94(6): 21712177. [crossref]

16. Zimering MB, Pulikeyil AT, Myers CE, Pang KC (2020) Serotonin 2A Receptor Autoantibodies Increase in Adult Traumatic Brain Injury In Association with Neurodegeneration. J Endocrinol Diabetes 7(1): 1. [crossref]

17. Shibata S, Sasaki T, Harpel P, Fillit H (1994) Autoantibodies to vascular heparan sulfate proteoglycan in systemic lupus erythematosus react with endothelial cells and inhibit the formation of thrombin-antithrombin III complexes. Clin Immunol Immunopathol 70: 114-123.

18. Peterson LK, Willis R, Harris EN, Branch WD, Tebo AE (2016) Antibodies to Phosphatidylserine/Prothrombin Complex in Antiphospholipid Syndrome: Analytical and Clinical Perspectives. Adv Clin Chem 73: 1-28. [crossref]

19. Zuo Y, Estes SK, Ali RA, Gandhi AA, Yalavarthi S, et al. (2020) Prothrombotic autoantibodies in serum from patients hospitalized with COVID-19. Sci Transl Med 12(570): eabd 3876

20. Heimer R, Klein G (1976) Circulating immune complexes in sera of patients with Burkett's lymphoma and nasopharyngeal carcinoma. Int J Cancer 18(3): 310-316. [crossref]

21. Zimering MB, Nadkarni SG, et al. (2019) Schizophrenia Plasma Autoantibodies Promote 'Biased Agonism' at the 5-Hydroxytryptamine 2A Receptor: Neurotoxicity is Positively Modulated by Metabotropic Glutamate 2/3 Receptor Agonism. Endocrinol Diabetes Metab J 3(4). [crossref]

22. Schmid CL, Raehal KM, Bohn LM (2008) Agonist-directed signaling of the serotonin 2A receptor depends on beta-arrestin-2 interactions in vivo. Proc Natl Acad Sci U S A 105(3): 1079-1084. [crossref]

23. Alsip NL, Harris PD, Durrani GE (1991) Multiple Serotonin Receptors on Large Arterioles in Striated Muscle. J Vasc Res 28: 537-541.

24. Pandey SC (2000) Cellular localization of serotonin (2A) (5HT (2A)) receptors in the rat brain. Brain Res Bull 51: 499-505. [crossref]

25. Zimering MB, Alder J, Pan Z, Donnelly RJ, et al. (2011) Anti-endothelial and antineuronal effects from auto-antibodies in subsets of adult diabetes having a cluster of microvascular complications. Diabetes Res Clin Pract 93(1): 95-105.

26. Zimering MB, Razzaki T, Tsang T, Shin JJ, et al. (2020) Inverse Association between Serotonin 2A Receptor Antagonist Medication Use and Mortality in Severe COVID-19 Infection. Endocrinol Diabetes Metab J 4(4): 1-5. [crossref] 